\title{
Alumina para utilização cerâmica, obtida a partir do rejeito de beneficiamento de caulim*
}

\section{(Alumina for ceramic manufacture using residues of kaolin processing)}

\author{
S. M. P. Flores, R. F. Neves \\ Departamento de Engenharia Química, Universidade Federal do Pará \\ C. P. 10110, 66.065-360, Belém, PA \\ e-mail:cpflores@interconect.com.br
}

\section{Resumo}

O beneficiamento de caulim para cobertura de papel produz grandes volumes de rejeito, constituído essencialmente do argilomineral caulinita, usado neste trabalho como material de partida para síntese de alúmen de amônio, visando a obtenção de alumina livre de sódio e de baixa granulometria para utilização cerâmica. O método de síntese desenvolvido para obtenção do alúmen de amônio constitui-se das etapas: calcinação do rejeito, lixiviação sulfúrica da metacaulinita, seguida da neutralização/cristalização da solução de $\mathrm{Al}_{2}\left(\mathrm{SO}_{4}\right)_{3}$ com NH $\mathrm{NH}_{4} \mathrm{OH}$ concentrado. No processo, são estudadas as variáveis: temperatura de calcinação $\left(650{ }^{\circ} \mathrm{C}, 700^{\circ} \mathrm{C}, 750{ }^{\circ} \mathrm{C}\right)$ e tempo de calcinação do rejeito (30,60 e 120 min), concentração de $\mathrm{H}_{2} \mathrm{SO}_{4}$, temperatura de lixiviação $\left(70{ }^{\circ} \mathrm{C}, 80{ }^{\circ} \mathrm{C}\right.$ e $\left.90{ }^{\circ} \mathrm{C}\right)$ sobre a cinética de lixiviação do alumínio. Estudou-se também o efeito do $\mathrm{pH}$ na cristalização do alúmen de amônio, apresentando-se as análises químicas, DRX, ATD, ATG e granulometria dos materiais utilizados e sintetizados.

\section{INTRODUÇÃO}

O processo de beneficiamento de caulim produz dois tipos de rejeitos: um, composto por partículas grosseiras (principalmente quartzo sob a forma de areia), que são repostas no próprio local da lavra, e um outro tipo de rejeito volumoso, constituído essencialmente por uma suspensão aquosa do argilomineral caulinita, sob a forma de partículas empilhadas, que não são totalmente dispersas durante o processamento, estando acompanhado de teores de ferro e titânio na ordem de 5\%.

$\mathrm{O}$ argilomineral caulinita, pertence ao grupo da candita [1], apresentando fórmula química (na forma de óxidos) $\mathrm{Al}_{2} \mathrm{O}_{3} \cdot 2 \mathrm{SiO}_{2} \cdot 2 \mathrm{H}_{2} \mathrm{O}$. Seus cristais possuem dimensões da ordem de $2 \mu \mathrm{m}$ ou inferiores, $\mathrm{o}$ que é vantajoso pois dispensa moagem para redução de sua granulometria. A estrutura cristalina de sua célula unitária é forma-

\footnotetext{
${ }^{(*)}$ Este trabalho é parte da tese de doutoramento da primeira autora, desenvolvido no Depto. de Química, CCEN, Universidade Federal do Pará; recebeu o Prêmio ALCOA no $41^{\circ}$ Congresso Brasileiro de Cerâmica, em $S$ Paulo, junho de 1997.
}

\section{Abstract}

The kaolin processing for paper covering produces a great volume of residues formed primarily by kaolinite claymineral, here the starting material for the synthesis of ammonium alumen aiming to obtain free sodium alumina with fine grade for ceramic manufacturing. The synthesis process to obtain ammonium alumen consisted of the following procedures: residues calcination and sulphuric leaching of metakaolinite followed by neutralization/ crystallisation of aluminum sulphate solution with ammonium hydroxide concentrated solution. The effect of calcination temperature $\left(650{ }^{\circ} \mathrm{C}, 700{ }^{\circ} \mathrm{C}\right.$ and $\left.750{ }^{\circ} \mathrm{C}\right)$, calcination time of residues ( $30 \mathrm{~min}, 60 \mathrm{~min}$ and $120 \mathrm{~min}$ ), sulphuric acid concentration as well as leaching temperature $\left(70{ }^{\circ} \mathrm{C}, 80{ }^{\circ} \mathrm{C}\right.$ and $90{ }^{\circ} \mathrm{C}$ ) on the kinetics of aluminum leaching were studied. The influence of $\mathrm{pH}$ on ammonium alumen crystallization was also studied. Data on chemical analysis, XRD, DTA, TGA and granulometry of the material utilized and synthesized are presented and discussed.

da por uma folha de tetraedros de silício e oxigênio e outra, octaédrica, composta de alumínio, oxigênio e hidroxila, unindo-se as mesmas por oxigênios comuns às duas folhas $[1,2]$.

O objetivo deste trabalho é apresentar resultados preliminares sobre o aproveitamento do rejeito de beneficiamento de caulim como fonte de alumínio, para produção de alúmem de amônio, que é utilizado como precursor de alumina livre de sódio e com baixa granulometria, para utilização cerâmica.

\section{MATERIAIS E MÉTODOS}

A matéria-prima utilizada como fonte para obtenção do alumínio foi o rejeito originado no processo de beneficiamento do caulim Amazon-88, produzido pela Caulim da Amazônia CADAM, fornecido pela referida empresa.

$\mathrm{O}$ ácido sulfúrico utilizado para o processo de lixiviação foi uma solução aquosa de ácido sulfúrico $(3,5 \mathrm{~N})$ em quantidades que proporcionassem as relações alumínio/ácido iguais a estequiométrica e $10 \%$ acima da estequiométrica, em função do teor de alumínio 
presente no rejeito e de modo a permitir a formação de uma solução de sulfato de alumínio na concentração máxima de $200 \mathrm{~g} / \mathrm{L}$.

O hidróxido de amônio utilizado para a reação de cristalização do alúmen de amônio foi hidróxido de amônio comercial concentrado (29\%).

A alumina utilizada como amostra de referência foi a alumina A-16SG produzida pela ALCOA (USA).

As análises por difração de raios $\mathrm{X}$ foram realizadas empregando-se o método do pó, com difratômetro tipo PW3710 BASED.

As análises termogravimétrica (ATG) e termodiferencial (ATD) foram realizadas em equipamento Derivatograph MOM, com velocidade de aquecimento constante de $20{ }^{\circ} \mathrm{C} / \mathrm{min}$ até $1200{ }^{\circ} \mathrm{C}$.

A granulometria foi determinada em sedígrafo Mastersizer/E da Malvern e a área específica (BET) em aparelho Monosorb Quantachrome.

A análise química do rejeito foi realizada por via úmida. As determinações dos teores de $\mathrm{Al}$ e Fe extraídos na lixiviação ácida foram realizadas empregando-se os métodos titulométrico com EDTA e dicromatométrico, respectivamente. A análise química da alumina foi feita pelo método da fluorescência de raios X.

As calcinações foram realizadas em forno mufla de resistência elétrica nas temperaturas de 650,700 e $750{ }^{\circ} \mathrm{C}$.

A lixiviação ácida realizou-se em reator de vidro de três vias sob agitação e sob refluxo, com termômetro e termostato acoplados e aquecido em manta elétrica.

\section{MÉTODO DE OBTENÇÃO DO ALUMEN DE AMÔNIO.}

O método de síntese consta das seguintes etapas:

\section{Calcinação do rejeito}

Ataques ácidos diretos de argilas cauliníticas para solubilização do Al não são eficazes. No entanto, após tratamento térmico adequado, ocorre desidroxilação com transformação da rede cristalina (formação da metacaulinita), tornando o material susceptível ao ataque ácido. Todavia, temperaturas elevadas $\left(>800{ }^{\circ} \mathrm{C}\right)$ são capazes de tornálo novamente insolúvel devido à formação de mulita [1-3].

\section{Lixiviação sulfúrica da metacaulinita}

A metacaulinita pode ser facilmente lixiviada empregando-se $\mathrm{H}_{2} \mathrm{SO}_{4}$ em temperaturas próximas de 70 a $90{ }^{\circ} \mathrm{C}$ e pressão atmosférica, sendo que além de $\mathrm{Al}$, Fe e pequenas quantidades de $\mathrm{Si}$ são co-extraídos durante o processo [3]. Nesta etapa obtém-se $\mathrm{Al}_{2}\left(\mathrm{SO}_{4}\right)_{3}$ em solução.

\section{Neutralização / Cristalização}

A solução de sulfato de alumínio, contendo excesso de $\mathrm{H}_{2} \mathrm{SO}_{4}$ livre para prevenir a hidrólise dos sais de ferro [4], é reagida lentamente com $\mathrm{NH}_{4} \mathrm{OH}$ concentrado até $\mathrm{pH}=0,00$, para formação do alúmen de amônio, segundo a reação simplificada proposta na equação (A).

$$
\mathrm{Al}_{2}\left(\mathrm{SO}_{4}\right)_{3}+\mathrm{H}_{2} \mathrm{SO}_{4}+2 \mathrm{NH}_{4} \mathrm{OH}+22 \mathrm{H}_{2} \mathrm{O} \rightarrow\left(\mathrm{NH}_{4}\right)_{2} \mathrm{SO}_{4} \cdot \mathrm{Al}_{2}\left(\mathrm{SO}_{4}\right)_{3} \cdot 24 \mathrm{H}_{2} \mathrm{O} \downarrow
$$

\section{OBTENÇÃO DA ALUMINA}

A alumina é obtida a partir da calcinação do alúmen de amônio sintetizado, passando pelas seguintes transformações [5], até a temperatura de $1200{ }^{\circ} \mathrm{C}$ :
$\mathrm{AlNH}_{4}\left(\mathrm{SO}_{4}\right)_{2} \cdot 12 \mathrm{H}_{2} \mathrm{O} \rightarrow \mathrm{AlNH}_{4}\left(\mathrm{SO}_{4}\right)_{2} \cdot 2 \mathrm{H}_{2} \mathrm{O} \rightarrow \mathrm{AlNH}_{4}\left(\mathrm{SO}_{4}\right)_{2} \rightarrow \mathrm{Al}_{2}\left(\mathrm{SO}_{4}\right)_{3} \rightarrow$ alumina amorfa $\rightarrow \gamma$-alumina $\rightarrow \delta$-alumina $\rightarrow \theta$-alumina $\rightarrow \alpha$-alumina.

\section{RESULTADOS E DISCUSSÃO}

\section{Composição química do rejeito}

A suspensão do rejeito foi seca a $110{ }^{\circ} \mathrm{C}$ por 24 horas e analisada, apresentando a composição indicada na Tabela I.

Tabela I: Composição química em \%, do rejeito de beneficiamento de caulim e do teórico da caulinita .

\begin{tabular}{lll}
\hline Óxido & $\begin{array}{l}\text { Rejeito de } \\
\text { Caulim }\end{array}$ & Caulinita \\
\hline $\mathrm{PF}$ & 13,35 & 13,96 \\
\hline $\mathrm{Al}_{2} \mathrm{O}_{3}$ & 35,63 & 39,50 \\
\hline $\mathrm{Si} \mathrm{O}_{2}$ & 41,82 & 46,54 \\
\hline $\mathrm{Fe}_{2} \mathrm{O}_{3}$ & 2,35 & - \\
\hline $\mathrm{TiO}_{2}$ & 2,25 & - \\
\hline $\mathrm{CaO}_{\mathrm{MgO}}$ & 0,18 & - \\
\hline $\mathrm{MgO}_{2}$ & 0,20 & - \\
\hline $\mathrm{Na}_{2} \mathrm{O}$ & 1,83 & - \\
\hline $\mathrm{K}_{2} \mathrm{O}$ & 1,25 & - \\
\hline $\mathrm{P}_{2} \mathrm{O}_{5}$ & 0,30 & \\
\hline
\end{tabular}

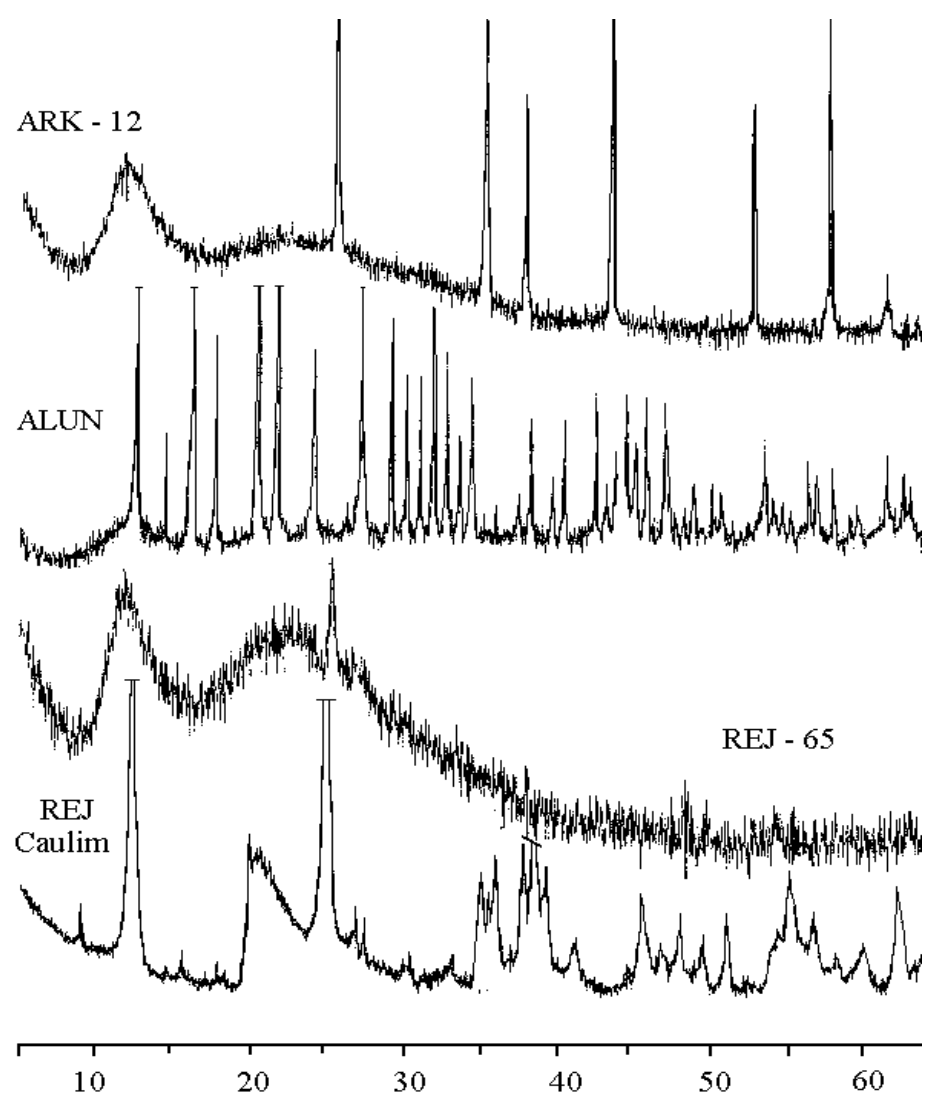

Figura 1: Difratograma de raios $X$ do rejeito utilizado, do rejeito calcinado, do alúmem sintetizado e da alumina obtida por calcinação do alúmen, a $1200{ }^{\circ} \mathrm{C}$. 


\section{Difração de Raios $X$}

A difração de raios $\mathrm{X}$ do rejeito (REJ) indicou a presença preponderante do argilomineral caulinita, com teores menores de anatase e, possivelmente, alguma crandalita; o rejeito calcinado (REJ-65), apresentou-se não cristalino, com a presença de picos de baixa intensidade de anatase; já o material obtido por síntese, constituiu-se essencialmente de alúmen de amônio (ALUN) e a alumina obtida por calcinação do alúmen apresentou-se como alumina alfa (ARK - 12), como mostrados na Fig. 1.

\section{Análises Térmicas (ATD - ATG)}

Análises termogravimétrica e termodiferencial do rejeito, indicaram o pico endotérmico a $570-580{ }^{\circ} \mathrm{C}$ característico de caulinita, com perda de massa de 13,8\%, referente à desidroxilição, por volta de $566{ }^{\circ} \mathrm{C}$ (formação da metacaulinita), muito próximo do valor teórico $(13,96 \%)$, e um pico exotérmico a $994^{\circ} \mathrm{C}$, relativo à nucleação de mulita [1,2], conforme a Fig. 2 .

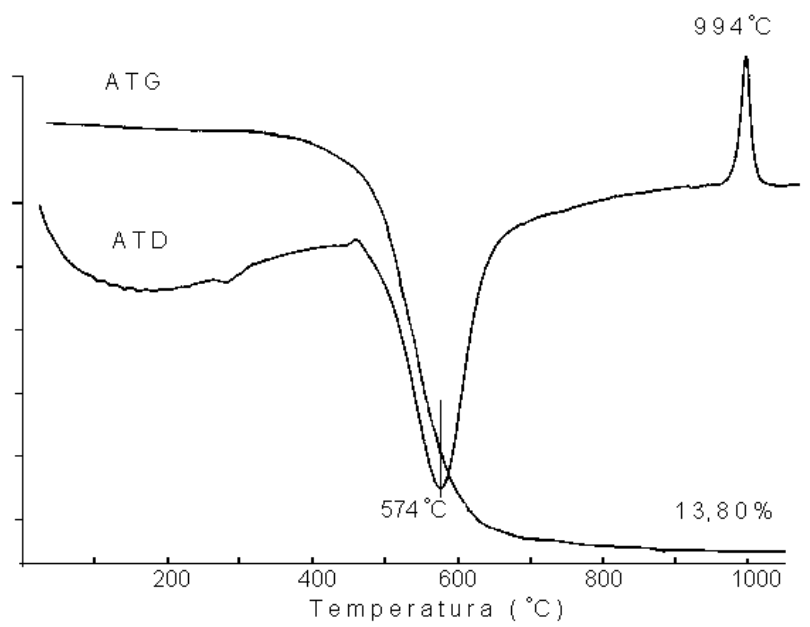

Figura 2: Análise térmica diferencial e termogravimétrica do rejeito de caulim (REJ).

\section{Calcinação do Rejeito}

Para o estabelecimento das temperaturas de calcinação do rejeito do caulim, utilizou-se, além das curvas de ATD e ATG, o desenvolvimento de ensaios preliminares, onde foram realizados testes padronizados de lixiviação com $\mathrm{H}_{2} \mathrm{SO}_{4}$, tomando-se como padrão a relação de $10 \%$ acima do estequiométrico referente à equação (B) para formação de sulfato de alumínio (relação $2 \mathrm{Al}: 3,3$ $\mathrm{H}_{2} \mathrm{SO}_{4}$ ) em função do teor total de alumínio presente no material, de modo também a se obter uma solução de $\mathrm{Al}_{2}\left(\mathrm{SO}_{4}\right)_{3}$ à $200 \mathrm{~g} / \mathrm{L}$, e excesso de ácido.

$$
\left(\mathrm{Al}_{2} \mathrm{O}_{3} .2 \mathrm{SiO}_{2}\right)+3 \mathrm{H}_{2} \mathrm{SO}_{4} \rightarrow \mathrm{Al}_{2}\left(\mathrm{SO}_{4}\right)_{3}+2 \mathrm{SiO}_{2}+3 \mathrm{H}_{2} \mathrm{O}
$$

Os parâmetrosanalisados foramos efeitos de temperatura de calcinação (Tc) que foram $650,700 \mathrm{e} 750{ }^{\circ} \mathrm{C}$, durante $120 \mathrm{~min}$ e, para a temperatura de $650^{\circ} \mathrm{C}$, variou-se o tempo de calcinação em 30,60 e $120 \mathrm{~min}$.

Nas Tabelas II e III são mostrados os rendimentos de dissolução do alumínio em função das temperaturas e do tempo de calcinação a que o rejeito foi submetido.
Tabela II: Rendimento da lixiviação do alumínio do rejeito de caulim, em função da temperatura de calcinação.

\begin{tabular}{llll}
\hline Tempo & \multicolumn{4}{c}{ Temperatura $\left({ }^{\circ} \mathbf{C}\right)$} \\
\hline minutos & 650 & 700 & 750 \\
\hline 120 & $94,14 \%$ & $88,92 \%$ & $50,51 \%$ \\
\hline
\end{tabular}

Tabela III: Rendimento da lixiviação do alumínio do rejeito de caulim calcinado a $650^{\circ} \mathrm{C}$, em função do tempo de calcinação.

\begin{tabular}{llll}
\hline Temperatura & \multicolumn{3}{c}{ Tempo (min) } \\
\hline${ }^{\circ} \mathrm{C}$ & 30 & 60 & 120 \\
\hline 650 & $93,07 \%$ & $96,72 \%$ & $94,14 \%$ \\
\hline
\end{tabular}

\section{Lixiviação Sulfúrica}

Com base nos resultados das Tabelas II e III, foram escolhidos os parâmetros a serem utilizados nos testes de lixiviação, como sendo: temperatura de calcinação $(\mathrm{Tc})=650{ }^{\circ} \mathrm{C}$ e tempo de calcinação (tc) $=60 \mathrm{~min}$, nas condições de lixiviação padrão, com $10 \%$ de $\mathrm{H}_{2} \mathrm{SO}_{4}$ acima do estequiométrico.

Nesta etapa, o parâmetro estudado foi a temperatura de lixiviação (Tl), 70, 80 e $90{ }^{\circ} \mathrm{C}$ em função da cinética da lixiviação do alumínio, tendo-se constatado que o tempo e a temperatura de lixiviação mais adequados foram $120 \mathrm{~min}$ e $90^{\circ} \mathrm{C}$, respectivamente.

Foram também realizados ensaios, onde estudou-se a redução da concentração do $\mathrm{H}_{2} \mathrm{SO}_{4}$ para uma proporção correspondente ao valor estequiométrico, para formação de sulfato de alumínio (relação $2 \mathrm{Al} \mathrm{:} 3 \mathrm{H}_{2} \mathrm{SO}_{4}$ ), em função do teor total de alumínio presente na amostra. Na Tabela IV, apresentam-se os valores de dissolução do alumínio por lixiviação.

Tabela IV: Rendimento da lixiviação do alumínio do rejeito de caulim, em função da temperatura de lixiviação e da concentração do ácido utilizado, após 120 min de lixiviação.

\begin{tabular}{lllc}
\hline Conc. do Ácido & \multicolumn{4}{l}{ Temperatura de Lixiviação $\left({ }^{\circ} \mathbf{C}\right)$} \\
& 70 & 80 & 90 \\
\hline $10 \%>$ Esteq. & $67,49 \%$ & $88,23 \%$ & $96,72 \%$ \\
\hline Esteq. & - & - & $95,09 \%$ \\
\hline
\end{tabular}

Esteq. - Valor estequiométrico necessário para formação de $\mathrm{Al}_{2}\left(\mathrm{SO}_{4}\right)_{3}$.

\section{Neutralização / Cristalização}

Pesquisas anteriores [6-8] mostram que o alúmen de amônio obtido por esse método forma-se somente quando há em solução um elevado excesso de ácido, suficiente para baixar o $\mathrm{pH}$ abaixo 


\section{Neutralização / Cristalização}

Pesquisas anteriores [6-8] mostram que o alúmen de amônio obtido por esse método forma-se somente quando há em solução um elevado excesso de ácido, suficiente para baixar o $\mathrm{pH}$ abaixo de zero e que, ao adicionar-se $\mathrm{NH}_{4} \mathrm{OH}$ à essa solução, atingindo-se $\mathrm{pH}$ zero, obtém-se uma rápida cristalização do alúmen de amônio. Continuando a adição de $\mathrm{NH}_{4} \mathrm{OH}$, o pH eleva-se e o alúmen formado vai-se transformando em $\mathrm{Al}(\mathrm{OH})_{3}$ gelatinoso. De acordo com o exposto, tomou-se $100 \mathrm{~mL}$ da solução que obteve $96,72 \%$ de eficiência na extração do $\mathrm{Al}$, adicionou-se um excesso de $6,77 \mathrm{~mL}$ de $\mathrm{H}_{2} \mathrm{SO}_{4}$ (até $\mathrm{pH}=0,70)$ e deixou-se reagir com $\mathrm{NH}_{4} \mathrm{OH}$ concentrado até $\mathrm{pH}=0,00$, sendo gastos para isso $14,6 \mathrm{~mL}$ de $\mathrm{NH}_{4} \mathrm{OH}$, valor esse muito próximo do teórico (13,76 mL). A solução com o precipitado, ficou em repouso $70 \mathrm{~h}$ para crescimento dos cristais, sendo então filtrada a vácuo. Os cristais foram lavados com etanol e deixados secar em dessecador. O rendimento em alúmen de amônio foi de $96,13 \%$. Os cristais obtidos foram analizados por difração de raios $\mathrm{X}$, confirmando-se assim a formação do alúmen de amônio, como pode ser visto na Fig. 1.

\section{Calcinação}

O alúmen de amônio sintetizado, foi calcinado em forno de maçarico a $1200 \pm 10^{\circ} \mathrm{C}$ por $1 \mathrm{~h}$, obtendo-se uma alumina esponjosa, facilmente desagregavel, cuja análise por difração de raios $\mathrm{X}$ encontra-se na Fig. 1. A análise química da alumina produzida nesta pesquisa, a ARK-12, realizada por fluorescência de raios X, está apresentada na Tabela $\mathrm{V}$, onde também consta a composição da alumina de referência A-16SG (ALCOA-USA), as quais apresentaram teores de $\mathrm{Al}_{2} \mathrm{O}_{3}$ alfa de $100 \%$ e $74,18 \%$, respectivamente.

O rendimento em alumina, foi de $92,79 \%$, em relação ao alumínio contido no rejeito (REJ).

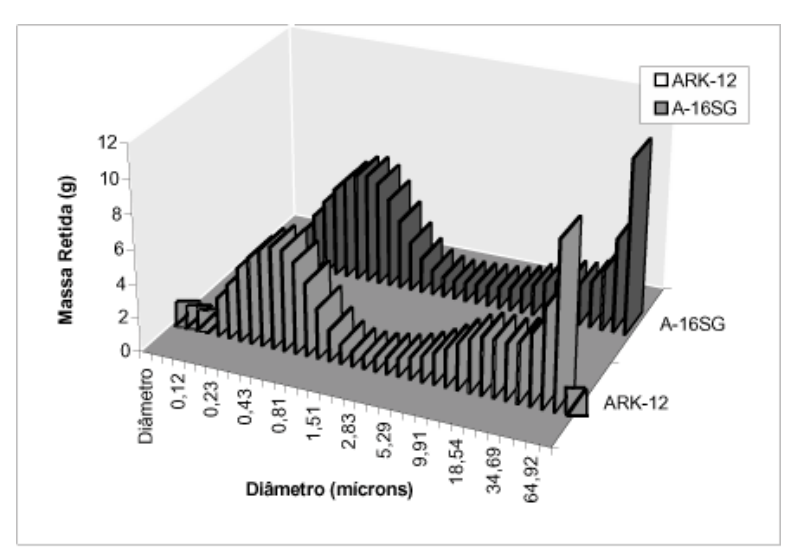

Figura 3: Distribuição granulométrica da alumina sintetizada ARK-12 e da alumina A-16SG da ALCOA(USA).

\section{Moagem}

A alumina sintetizada (ARK-12), foi moída a seco por $60 \mathrm{~h}$ em moinho de bolas de laboratório, apresentando granulometria conforme demonstrada na Fig. 3, onde também aparece a distribuição granulométrica da alumina utilizada como referência (A-16SG da ALCOA-USA), para efeito de comparação.
Tabela V: Composição química da alumina sintetizada e da alumina de referência.

\begin{tabular}{lll}
\hline Óxidos $(\%)$ & ARK-12 & A-16SG \\
\hline $\mathbf{F e}_{\mathbf{2}} \mathbf{O}_{\mathbf{3}}$ & 0,553 & 0,0120 \\
\hline $\mathbf{S i O}_{\mathbf{2}}$ & 0,135 & 0,0240 \\
\hline $\mathbf{T i O}_{2}$ & $<0,001$ & $<0,0010$ \\
\hline $\mathbf{Z n O}$ & 0,001 & 0,0020 \\
\hline $\mathbf{V}_{\mathbf{2}} \mathbf{O}_{\mathbf{5}}$ & $<0,001$ & $<0,0010$ \\
\hline $\mathbf{C a O}$ & 0,001 & 0,0210 \\
\hline $\mathbf{G a}_{\mathbf{2}} \mathbf{O}_{\mathbf{3}}$ & 0,009 & 0,0030 \\
\hline $\mathbf{N a}_{\mathbf{2}} \mathbf{O}$ & 0,00 & 0,0700 \\
\hline $\mathbf{P}_{\mathbf{2}} \mathbf{O}_{\mathbf{5}}$ & 0,0321 & 0,0008 \\
\hline $\mathbf{S O}$ & $<0,001$ & 0,0100 \\
\hline $\mathbf{M n O}$ & 0,0005 & 0,0006 \\
\hline
\end{tabular}

\section{CONCLUSÕES}

A metodologia de produção de alúmen de amônio através da utilização do rejeito de beneficiamento de caulim é tecnicamente viável, segundo os parâmetros de calcinação, lixiviação e neutralização/cristalização adotados nesta pesquisa.

A etapa de neutralização / cristalização do processo, deverá ser realizada após a adição à solução da extração, de um excesso de $87 \%$ de ácido sulfúrico, em relação ao volume usado na extração, o que significa uma relação molar de $2 \mathrm{Al}: 5,6 \mathrm{H}_{2} \mathrm{SO}_{4}$, sendo que esta adição, necessária apenas nesta etapa, não acarreta os inconvenientes do manuseio de soluções muito concentradas, até que se atinja esta fase do processo.

A alumina sintetizada é isenta de sódio e, embora apresente uma distribuição granulométrica semelhante à da alumina empregada como parâmetro de referência, a A-16SG, sua área específica, substancialmente mais elevada do que desta última, parece indicar ainda a presença de aglomerados não destruídos na moagem, sendo necessárias outras análises para permitir conclusões mais concretas a esse respeito.

\section{REFERÊNCIAS}

[1] P. Souza Santos, Tecnologia de argilas, aplicada às argilas brasileiras, São Paulo, S.P, Edgard Blucher V1,(1975).

[2] R. E. Grim, Clay Mineralogy, 2.ed. N. York McGraw Hill, (1968). [3] A. L. M. Carneiro, Fontes alternativas de obtenção de alfaalumina. Ensaios de calcinação e lixiviação em escala de laboratório. In: II Encontro do Hemisfério Sul sobre Tecnologia Mineral. Anais, V2. R. de Janeiro (1987) p. 219-234.

[4] E. A. Gee, W. K. Cunningham, R. A. Heindl, Production of iron-free alum. Industrial and Engineering Chemistry 39, 9 (1947) 1178-1188.

[5] T. Sato, F. Ozawa, S. Ikoma, Thermal decomposition of 
aluminium salts - hydrates of the chloride, nitrate and sulphate, and of ammonium alum. J. Appl. Chem. Biotechnol 28, 12 (1978) 811-822.

[6] J. M. R. Mercury, Características cerâmicas de aluminas obtidas através da reação de sulfato de alumínio e hidróxido de amônio: Efeito da temperatura de síntese, Dissertação de Mestrado em Engenharia Química, DEQ - UFPA (1995).
[7] M. C. Martinelli, R. F. Neves, Obtenção de alúmen de amônio como precursor de alumina para cerâmica através da reação de sulfato de alumínio e hidróxido de amônio. Cerâmica 42, 274 (1996).

[8] S. M. P. Flores, M. N. S. Castro, J. H. B. Costa, R. F. Neves, Obtenção de alúmen de amônio, a partir do rejeito de beneficiamento de caulim. Cerâmica 42, 274, (1996) 51.

(Rec. 30/10/97, Ac. 24/11/97). 